\title{
Peut-on parler d'optimisation de la radioprotection dans les domaines médical et industriel non électronucléaire en France? *
}

\author{
C. LEFAURE**, G. ABADIA***, B. AUBERT****
}

(Manuscrit reçu le 19 décembre 1994, accepté le 19 janvier 1995)

RÉSUMÉ En 1993, plus de 10000 travailleurs, soit près du quart des travailleurs exposés de façon significative, travaillent dans les domaines médical, de la recherche et des industries non électronucléaires en France. La dose moyenne annuelle par travailleur significativement exposé est de $2 \mathrm{mSv}$ pour les vétérinaires, $3 \mathrm{mSv}$ pour les travailleurs du secteur médical, $5,5 \mathrm{mSv}$ pour les travailleurs de l'industrie non électronucléaire. Dans le secteur industriel, plus de $8 \%$ des travailleurs exposés ont une exposition annuelle supérieure à $15 \mathrm{mSv}$. Ces expositions ne sont ni négligeables, ni véritablement plus faibles que celles observées dans l'électronucléaire où l'optimisation de la radioprotection est couramment appliquée. A ce jour, aucune publication ne fait état d'expériences en matière d'optimisation dans les autres secteurs. La réalisation d'études d'optimisation dans des centres de référence, pour des examens radiologiques ou des pratiques industrielles spécifiques, en particulier pour toutes les nouvelles pratiques telles que la radiologie interventionnelle, permettrait de fournir aux autres entreprises, services ou cabinets, des informations sur ce que pourrait être une pratique optimisée, dans des conditions de référence, en matière de radioprotection. Les vraies difficultés pour la mise en auvre de l'optimisation dans les secteurs industriel et médical ne sont pas celles le plus souvent mises en avant (faibles niveaux d'exposition, ressources insuffisantes, complexité...), mais plutôt un manque de données appropriées et surtout une absence de culture du risque résiduel.

ABSTRACT In France, in 1993, more than 10000 workers, i.e. nearly a quarter of the workers significantly exposed, were working in the medical, research and nonnuclear industrial fields. The annual average dose per significantly exposed worker was $2 \mathrm{mSv}$ for veterinary surgeons, $3 \mathrm{mSv}$ for the medical sector, $5.5 \mathrm{mSv}$ for the non-nuclear industry. In the industrial sector, more than $8 \%$ of the exposed workers received an annual dose higher than $15 \mathrm{mSv}$. Such exposures are neither negligible nor really lower than those of the nuclear industry where the optimisation of radiological protection is currently implemented. So far, no publication has mentioned experience in the other sectors. Optimisation surveys in reference centres, for $x$-ray examinations or specific industrial prac-

\footnotetext{
* Communication présentée lors des journées SFRP, “L'optimisation de la radioprotection des travailleurs dans les domaines électronucléaire, industriel et médical", La Rochelle, 2021 septembre 1994.

** Centre d'étude sur l'évaluation de la protection dans le domaine nucléaire (CEPN), BP 48, 92263 Fontenay-aux Roses Cedex, France.

*** Institut national de la recherche pour la sécurité (INRS), 30 rue Olivier Noyer, 75014 Paris, France.

**** Institut Gustave Roussy, 94805 Villejuif Cedex, France.
} 
tices, especially for the new practices such as interventional radiology, would make it possible to supply other enterprises or radiological services with information on what an optimised practice might be, under reference conditions, as far as radiological protection is concerned. The true difficulties in the implementation of optimisation in the industrial and medical sectors are not those which are commonly put forward (low levels of exposure, lack of resources, complexity...) but rather a lack of relevant data and above all a lack of culture on the residual risk.

\section{Introduction}

Un décret du 2 octobre 1986 [10] pour l'ensemble des installations non électronucléaires, et un autre du 6 mai 1988 [11] pour les installations nucléaires précisent que le principe d'optimisation de la radioprotection doit être appliqué dans toutes les installations utilisant des rayonnements ionisants, sans exception. Les travailleurs exposés aux rayonnements ionisants peuvent être regroupés en quatre secteurs différents : le cycle électronucléaire, le secteur médical où les rayonnements sont utilisés à des fins diagnostiques et thérapeutiques, le secteur industriel non électronucléaire où les sources de rayonnements correspondent à des applications très diverses (contrôles non destructifs, stérilisation, conservation, mesures de niveau, mesures d'humidité...) et le secteur de la recherche, souvent plus ou moins confondu avec les secteurs précédents.

Dans le secteur électronucléaire, l'optimisation est maintenant devenue une pratique courante, comme en témoignent les nombreux exposés présentés à ces journées. Par contre, à ce jour, aucune publication ne fait état d'expériences en matière d'optimisation dans les autres secteurs en France. Les arguments qui sont le plus souvent avancés pour justifier cette absence d'optimisation dans les secteurs industriels et médicaux sont : le faible niveau des expositions ("à quoi l'optimisation pourrait-elle servir, puisque l'on respecte largement les limites" ?) ; le manque de ressources ("l'optimisation est une démarche pour l'industrie électronucléaire qui en a les moyens") ; la complexité de la démarche et des techniques d'optimisation ("pour faire de l'optimisation il faut des spécialistes") ; la multiplicité des centres de décision ("sans volonté politique affirmée par une structure centralisée comment faire de l'optimisation" ?). De tels arguments, dont nous allons analyser la pertinence, ne permettent pas, à notre avis, de justifier une réelle absence de mise en œuvre de l'optimisation dans les secteurs industriel et médical. L'analyse de la situation dans ces secteurs montre, par contre, que les principaux obstacles sont le manque de données adéquates et surtout l'absence d'une véritable culture du risque résiduel aux faibles expositions. 


\section{Les expositions}

L'argument généralement avancé en premier pour justifier le peu d'intérêt à mettre en œuvre l'optimisation dans les domaines médical et industriel non électronucléaire ${ }^{1}$ est celui du niveau des expositions qui est suffisamment faible pour que des efforts supplémentaires paraissent superflus. Pour répondre à cet argument, il convient d'analyser les données disponibles, sachant qu'il n'existe pas, en France, de statistiques nationales, exhaustives et publiées concernant les expositions professionnelles aux rayonnements ionisants.

La dosimétrie réglementaire (film dosimètre) est réalisée par le Service central de protection contre les rayonnements ionisants (SCPRI devenu OPRI en juillet dernier), le Laboratoire central des industries électriques (LCIE), la société Philips pour les travailleurs du médical, de l'industrie et de la recherche et par les exploitants nucléaires pour leur propre personnel. En ce qui concerne l'année 1993, pour la première fois ${ }^{2}$, le SCPRI a regroupé ses données avec celles du LCIE et de Philips. Ces données réglementaires sont les seules disponibles pour les secteurs industriel et médical, contrairement au secteur électronucléaire où les exploitants disposent des données issues de la dosimétrie opérationnelle (dosimètres électroniques). Ces dernières constituent une source d'information fournissant une assez bonne connaissance en temps quasi réel des expositions individuelles et collectives (par tâche, zone,...) pour leur personnel et celui des entreprises contractantes. Enfin, il faut signaler que, si l'on excepte le cas des mineurs d'uranium, les doses enregistrées et accessibles ne concernent que les expositions externes, à l'exclusion de toutes celles liées à la contamination.

\subsection{Expositions collectives et individuelles moyennes}

A partir de ces différentes sources, on peut estimer, en 1993, que le nombre total de travailleurs suivis est supérieur à 210000 en France (Tab. I) répartis dans environ 20000 établissements. Par ailleurs, le nombre de travailleurs exposés de façon significative ${ }^{3}$ aux rayonnements ionisants, au sein de la population suivie, dépasse 43000 , dont un quart travaille hors du secteur électronucléaire.

Une approche purement statistique, à partir des données disponibles, montre que la dose moyenne annuelle 4 par travailleur significativement exposé est de 1 à $2 \mathrm{mSv}$ pour les employés des exploitants nucléaires (mineurs exclus)

1. Dans ce texte, on regroupera sous l'appellation "secteur industriel", les industries non électronucléaires et la recherche.

2. Il est à noter que, jusqu'à présent, les données fournies à l'UNSCEAR ou à la CCE par le SCPRI correspondaient aux seuls établissements directement suivis par ce dernier.

3. Les seuils de significativité de la dosimétrie réglementaire sont $0,2 \mathrm{mSv}$ pour le film mènsuel pour le SCPRI et EDF, $0,35 \mathrm{mSv}$ pour le groupe CEA.

4. Dans tout l'exposé, il s'agira de la dose "corps entier". 
TABLEAU I

Expositions collectives et individuelles moyennes par secteur en France en 1993 Mean collective and individual exposures in various fields, France, 1993

\begin{tabular}{|c|c|c|c|c|c|}
\hline Secteur & $\begin{array}{l}\text { Electron } \\
\text { dont ent. }\end{array}$ & $\begin{array}{l}\text { éaire } \\
E D F \\
(1)\end{array}$ & $\begin{array}{l}\text { Médical } \\
(2)\end{array}$ & $\begin{array}{c}\text { Recherche } \\
\text { et autres } \\
\text { industries } \\
(2)\end{array}$ & Total \\
\hline $\begin{array}{l}\text { Nombre de travail- } \\
\text { leurs surveillés }\end{array}$ & 79668 & 28765 & 103098 & 27183 & 211847 \\
\hline $\begin{array}{l}\text { Nombre de travail- } \\
\text { leurs exposés }\end{array}$ & 33099 & 14769. & $7745(4)$ & $2473(4)$ & 43438 \\
\hline $\begin{array}{l}\text { Dose collective } \\
\text { (homme.sievert) }\end{array}$ & 124,4 & 88,9 & 22,2 & 13,52 & 160,35 \\
\hline $\begin{array}{l}\text { Dose ind. moyenne } \\
\text { par travailleurs exposé } \\
\text { (mSv/an) }\end{array}$ & 3,76 & 6,02 & 2,87 & 5,47 & 3,69 \\
\hline
\end{tabular}

(1) Colonne calculée à partir de données fournies par les exploitants $[4,7]$.

(2) Colonne estimée à partir de données fournies par le SCPRI [19].

(3) Electronucléaire + médical + vétérinaires + autres industries et recherche.

(4) En appliquant au nombre total de travailleurs surveillés, le ratio "travailleurs exposés" / "travailleurs surveillés" observé sur la population suivie par le seul SCPRI.

et les vétérinaires, $3 \mathrm{mSv}$ pour les travailleurs du secteur médical, $5,5 \mathrm{mSv}$ pour les travailleurs de l'industrie non électronucléaire, $6 \mathrm{mSv}$ pour les travailleurs des entreprises contractantes des centrales nucléaires et environ $9 \mathrm{mSv}$ pour les mineurs d'uranium.

\subsection{Distribution des expositions individuelles}

Les valeurs précédentes étant des moyennes, il est intéressant d'analyser, dans la mesure des indicateurs disponibles, la distribution des expositions individuelles autour de ces moyennes. Elles peuvent, en effet, correspondre à des situations très homogènes ou bien, au contraire, cacher des disparités comme celles que l'on observe dans le secteur électronucléaire où la dose moyenne d'un calorifugeur d'entreprise extérieure $(12 \mathrm{mSv})$ est nettement plus élevée que celle d'un électricien-automaticien d'EDF (1 mSv).

Les données disponibles (Tab. II) montrent que moins de $20 \%$ du nombre de personnes excédant $15 \mathrm{mSv}$ travaillent dans les secteurs médical et de l'industrie non électronucléaire, alors que ces secteurs regroupent $24 \%$ de 
TABLEAU II

Répartition par secteur du nombre de travailleurs ayant dépassé 15 et $50 \mathrm{mSv}$ en France en 1993

Distribution by fields of the number of workers exceeding 15 and $50 \mathrm{mSv}$ (France, 1993)

\begin{tabular}{|c|c|c|c|c|}
\hline \multirow{2}{*}{$\begin{array}{c}\begin{array}{c}\text { Catégorie } \\
\text { de travailleurs }\end{array} \\
\text { Secteur }\end{array}$} & \multicolumn{2}{|c|}{$\begin{array}{c}\text { Travailleurs dont la dose } \\
\text { est > à } 15 \mathrm{mSv}\end{array}$} & \multicolumn{2}{|c|}{$\begin{array}{l}\text { Travailleurs dont la dose } \\
\text { est > à } 50 \mathrm{mSv}\end{array}$} \\
\hline & Nombre & $\begin{array}{c}\text { \% par rapport } \\
\text { à l'ensemble des } \\
\text { travailleurs exposés }\end{array}$ & Nombre & $\begin{array}{l}\text { \% par rapport } \\
\text { à l'ensemble des } \\
\text { travailleurs exposés }\end{array}$ \\
\hline $\begin{array}{l}\text { Electronucléaire } \\
\text { dont ent. ext. }\end{array}$ & 1966 & 5,93 & 2 & 0,01 \\
\hline des centrales & 1731 & 11,7 & 2 & 0,01 \\
\hline Médical & 258 & 3,33 & 54 & 0,70 \\
\hline $\begin{array}{l}\text { Recherche et } \\
\text { autres industries }\end{array}$ & 202 & 8,17 & 14 & 0,57 \\
\hline Total & 2426 & 5,58 & 70 & 0,16 \\
\hline
\end{tabular}

l'ensemble des travailleurs exposés. Dans le secteur industriel, les travailleurs excédant $15 \mathrm{mSv}$ représentent plus de $8 \%$ du nombre de travailleurs exposés, alors qu'ils ne représentent que 3,3 et $5,9 \%$ des travailleurs exposés respectivement dans le secteur médical et le cycle électronucléaire. On peut aussi observer que les dépassements de limite enregistrés sont quasi exclusivement le fait des secteurs médical et industriel non électronucléaire. Le tableau III permet d'affiner l'analyse au sein des secteurs médical et industriel. Des informations plus détaillées sont fournies en annexe A sur le nombre de personnes surveillées et exposées, leurs expositions, la répartition de ces expositions par classe de dose.

Les doses individuelles annuelles moyennes par activité apparaissent plus dispersées dans le secteur industriel, où elles passent de $1,9 \mathrm{mSv}$ pour le travail sur les sources scellées à $7,2 \mathrm{mSv}$ pour les activités non spécifiées, que dans le secteur médical où elles ne dépassent pas $4,4 \mathrm{mSv}$ en médecine du travail. Dans les deux secteurs, les pourcentages de travailleurs exposés dépassant $15 \mathrm{mSv}$ sont très variables selon l'activité : entre $0,8 \%$ et $5,6 \%$ dans le secteur médical ; entre 1,9 et $11,4 \%$ dans le secteur industriel. De tels pourcentages ne sont donc pas négligeables pour certaines sous-populations. 


\section{TABLEAU III}

Expositions individuelles annuelles moyennes

et pourcentage de travailleurs exposés dépassant $15 \mathrm{mSv}$ en 1993 en France, selon les activités dans les secteurs médical et industriel Mean annual individual exposures and percent of exposed workers exceeding $15 \mathrm{mSv}$ in the medical and industrial fields (France, 1993)

\begin{tabular}{|c|c|c|c|c|c|}
\hline \multicolumn{3}{|c|}{ Secteur médical } & \multicolumn{3}{|c|}{ Secteur industriel } \\
\hline $\begin{array}{l}\text { Activité } \\
\text { in }\end{array}$ & $\begin{array}{l}\text { Dose } \\
\text { dividuelle } \\
\text { noyenne } \\
\text { mSingin) } \\
\text { (1) }\end{array}$ & $\begin{array}{c}15 \mathrm{mSv} \\
(2)\end{array}$ & Activité & $\begin{array}{c}\text { Dose } \\
\text { individuelle } \\
\text { moyenne } \\
\text { (mSv/an) } \\
(1)\end{array}$ & $\begin{array}{c}>15 \mathrm{mSv} \\
(2)\end{array}$ \\
\hline $\begin{array}{l}\text { Radiodiagnostic: } \\
\text { - Hôpital } \\
\text { - Privé (1) } \\
\text { - Dentaire } \\
\text { - Médecine } \\
\text { du travail }\end{array}$ & $\begin{array}{l}3,9 \\
2,5 \\
2,0 \\
4,4\end{array}$ & $\begin{array}{l}5,6 \% \\
2,4 \% \\
2,2 \% \\
\\
5,6 \%\end{array}$ & $\begin{array}{l}\text { Radiographie } \\
\text { et gammagraphie } \\
\text { industrielle }\end{array}$ & 3,8 & $6,2 \%$ \\
\hline $\begin{array}{l}\text { Radiothérapie: } \\
\text { - Externe } \\
\text { - Curiethérapie }\end{array}$ & $\begin{array}{l}3,2 \\
3,5\end{array}$ & $\begin{array}{l}0,8 \% \\
4,9 \%\end{array}$ & $\begin{array}{l}\text { Sources non scellées } \\
\text { inustrie et recherche }\end{array}$ & 1,9 & $1,9 \%$ \\
\hline $\begin{array}{l}\text { Médecine nucléaire : } \\
\text { - in vivo } \\
\text { - in vitro }\end{array}$ & $\begin{array}{l}2,0 \\
1,6\end{array}$ & $1,6 \%$ & $\begin{array}{l}\text { Autres applications } \\
\text { non médicales } \\
\text { non ventilées }\end{array}$ & $\begin{array}{l}3,6 \\
7,2\end{array}$ & $\begin{array}{r}3,8 \% \\
11,4 \%\end{array}$ \\
\hline Total & $\begin{array}{l}2,9 \\
(3)\end{array}$ & $\begin{array}{c}3,3 \% \\
(3)\end{array}$ & Total & $\begin{array}{l}5,5 \\
(3)\end{array}$ & $\begin{array}{l}\mathbf{8 , 2} \% \\
(3)\end{array}$ \\
\hline
\end{tabular}

(1) Il s'agit de la dose moyenne annuelle 1993 par travailleur exposé, calculée sur les populations surveillées par le SCPRI.

(2) Pourcentage de travailleurs exposés à plus de $15 \mathrm{mSv}$ en 1993 ; ratio calculé sur les populations surveillées par le SCPRI.

(3) Valeurs pour l'ensemble des populations surveillées par le SCPRI, le LCIE et Philips.

\subsection{Sous-estimation du nombre de travailleurs exposés}

Une enquête sur les rayonnements ionisants, réalisée à l'initiative du ministère du travail par sondage dans la région Aquitaine, a été publiée dans le Bilan annuel sur les conditions de travail du Conseil supérieur de prévention des risques professionnels en 1993 [6]. Cette enquête a été effectuée par les inspecteurs du travail, et a porté sur 128 entreprises. Certains résultats de cette 
enquête sont décrits en détail dans l'annexe B. Pour notre objet, il en ressort essentiellement une sous-estimation du nombre de travailleurs exposés, en effet :

- d'une part, seuls $2 / 3$ des employeurs déclarant employer des travailleurs de catégorie A (personne directement affectée à des travaux sous rayonnements, et dont les conditions de travail sont susceptibles d'entraîner le dépassement des 3/10 èmes des limites annuelles), les font suivre dosimétriquement, ce qui conduit, a minima, à une sous-estimation du nombre de travailleurs de cette catégorie, voire du nombre de travailleurs réellement exposés, si ces travailleurs dépassent le seuil des $0,2 \mathrm{mSv}$ mensuels ;

- d'autre part, parmi les entreprises qui utilisent des sources, moins de $50 \%$ des entreprises industrielles, $72 \%$ des services médicaux et $35 \%$ des dentistes, déclarent des travailleurs de catégorie A ; ce faible pourcentage peut paraître surprenant, même si certaines activités ne justifient pas le port de dosimètres (sources très peu actives, risque d'exposition interne exclusif,...).

Il n'est évidemment pas possible de dire si les expositions des travailleurs non suivis dosimétriquement sont négligeables ou non, mais il est probable que certaines ne le soient pas et viennent alors accroître les valeurs présentées dans les points précédents (cf. $\S 1.1$ et 1.2 ).

\subsection{Conclusion}

$\mathrm{Au}$ vu de l'ensemble de ces informations, il apparaît donc que les expositions du personnel réellement exposé des secteurs médical et industriel ne sont ni négligeables, ni véritablement plus faibles que celles observées dans l'électronucléaire où l'optimisation est couramment appliquée. L'argument des faibles expositions ne peut être un "bon" argument en défaveur de l'optimisation. En réalité, utiliser cet argument revient implicitement, voire explicitement, à se référer au seul respect du principe de limitation de dose et à nier ou méconnaître la position de prudence face au risque résiduel aux faibles expositions [14].

\section{Le coût de la protection}

"Mettre en œuvre des actions de radioprotection en vue d'optimiser les niveaux d'exposition coûte cher, le secteur électronucléaire est riche, nous ne le sommes pas." Une telle réaction suppose que, compte tenu des ressources disponibles, la somme que l'on accepte de payer pour réduire le risque radiologique n'est pas la même dans tous les secteurs. Ceci reviendrait à dire que, pour un niveau de dose individuelle donné, la valeur de référence de l'hommesievert n'est pas la même dans ces divers secteurs. Or, dans un contexte national, ne serait-il pas normal que les moyens affectés à la réduction du risque radiologique soient identiques dans tous les secteurs ? Des écarts n'iraient-ils pas à l'encontre de l'équité, face au risque, prônée par la Commission interna- 
tionale de protection radiologique (CIPR) dans ses diverses publications et plus particulièrement la publication 60 [12] ?

Dire que les ressources ne sont pas identiques entre un secteur électronucléaire perçu comme "riche", des hôpitaux tributaires du déficit de la sécurité sociale, un secteur de la recherche dont les budgets diminuent et un secteur industriel non électronucléaire où se rencontrent tous les cas de figure, est un truisme. Mais qu'en est-il des montants affectés à la gestion du risque radiologique ? Nous disposons de très peu de données comparables à ce sujet, hormis quelques études réalisées au début des années 1980. Ces études montrent que, sur la base de diverses mesures adoptées à l'époque dans les réacteurs français, il est possible d'estimer en moyenne à 1,9 MF (ramenés en francs 1994) le coût implicite de l'homme-sievert évité [16], alors qu'à partir des mesures prises dans un service hospitalier bordelais, ce même coût implicite est estimé à 200 $\mathrm{kF}$ (ramenés en francs 1994) [17]. On pourrait en conclure qu'un rapport 10 existe entre les montants dépensés dans ces deux secteurs pour épargner un homme-sievert.

Cependant, ce rapport ne reflète-t-il pas en partie la disparité en matière de niveaux d'exposition entre les deux populations concernées ? En 1982, la dose annuelle moyenne dans le service médical étudié était inférieure à $1 \mathrm{mSv}$ quelle que soit la spécialité de travailleur concernée (radiologue, anesthésiste, préparateur...), sans grande dispersion entre les individus. Inversement, pour cette période, aucune statistique nationale n'est disponible à EDF pour les travailleurs des entreprises extérieures (DOSINAT n'est opérationnel que depuis 1993 [8]). Cependant, les données de grandes entreprises intervenant dans les centrales montrent des expositions individuelles moyennes d'environ $5 \mathrm{mSv}$ en 1983 avec des sous-populations atteignant presque $15 \mathrm{mSv}$. On peut alors comparer les coûts implicites observés à l'époque avec le système de valeurs monétaires de référence de l'homme-sievert dont EDF s'est doté depuis 1993 [8].

On constate que le rapport 10 entre les montants dépensés s'explique aisément dès lors que l'on tient compte du niveau des expositions individuelles moyennes annuelles. En effet, dans le service hospitalier on dépensait plus que ce qu'EDF dépenserait pour une population appartenant à cette classe d'exposition individuelle. Dans le cas étudié, le problème des ressources n'était donc pas un frein fondamental pour la mise en œuvre d'une procédure d'optimisation.

La généralisation de cette conclusion à l'ensemble du secteur n'est pas possible compte tenu du faible nombre d'informations disponibles. Elle paraît, cependant, plausible dans le secteur médical, compte tenu des mesures adoptées pour la conception et l'exploitation des services et cabinets médicaux. Par contre, dans le domaine industriel, nous ne disposons actuellement d'aucun exemple. 
TABLEAU IV

Comparaison des coûts implicites de l'homme-sievert évité par des actions de protection mises en ouvres dans "un" service hospitalier et dans les réacteurs au début des années 80 , avec les valeurs monétaires de référence d'EDF Comparison of the implicit costs of 1 man-Sv avoided by protection operations in one hospital department and in power plants in the early $80 \mathrm{~s} v \mathrm{~s}$. EDF reference monetary values

\begin{tabular}{|c|c|c|c|}
\hline & $\begin{array}{l}\text { Dose annuelle } \\
\text { moyenne } \\
(\mathrm{mSv})\end{array}$ & $\begin{array}{l}\text { Coût implicite par } \\
\text { homme-sievert } \\
\text { évité (kF 1994) }\end{array}$ & $\begin{array}{l}\text { Valeurs de référenc EDF en } \\
\text { fonction de la dose annuelle } \\
\text { moyenne de la population } \\
\text { considérée }\end{array}$ \\
\hline Service médical & $\approx 1$ & $200 \mathrm{kF}$ & $100 \mathrm{kF}$ \\
\hline \multirow[t]{2}{*}{ Réacteurs } & 5 & & $500 \mathrm{kF}$ \\
\hline & 15 & $1900 \mathrm{kF}$ & $2300 \mathrm{kF}$ \\
\hline
\end{tabular}

(1) Valeur pour une population ayant une dose annuelle approchant $5 \mathrm{mSv}$ en moyenne.

(2) Valeur pour une population ayant une dose annuelle approchant $15 \mathrm{mSv}$ en moyenne.

Les résultats du tableau doivent être maniés avec prudence, car ils ne concernent qu'un seul service hospitalier.

En conclusion de ce paragraphe, deux remarques peuvent être formulées :

- La valeur monétaire de base de l'homme-sievert, calculée à partir d'estimations de ce qu'une nation peut dépenser pour épargner un décès lié à des risques professionnels (cf. CIPR 22 [13]) ne peut, par définition, qu'être unique, indépendante de la catégorie professionnelle et de l'âge des populations concernées. La valeur de base de l'homme-sievert peut être estimée à environ $100 \mathrm{kF}$ en francs 1994 [14].

- Toute institution peut, en fonction de ses ressources et de ses objectifs propres (prix de l'acceptabilité sociale de son activité, "image"...), décider de dépenser plus que cette valeur de base, en particulier pour réduire en priorité les expositions professionnelles les plus élevées.

De plus, il convient de ne pas oublier que, dans un premier temps, l'optimisation revient très fréquemment à mettre en œuvre des pratiques qui, non seulement coûtent peu mais, en plus, permettent de réduire les coûts d'exploitation $[3,14]$. Cela est particulièrement vrai quand il s'agit d'organisation du travail en vue de réduire les temps d'exposition. Dans le domaine médical par exemple, plusieurs études américaines ont montré que, si des réductions de dose pour le personnel étaient obtenues par l'utilisation de protections (écrans, protège-seringues...), les améliorations les plus significatives résultaient surtout de changements dans la façon de travailler [9]. 


\section{La démarche "ALARA"}

"L'optimisation ne peut être faite que par des spécialistes, compte tenu de la complexité des techniques à mettre en œuvre". Cet argument cache un “vrai" faux problème. En effet, il n'est besoin ni d'être ingénieur ni d'être économiste pour faire de l'optimisation. D'un point de vue méthodologique, l'optimisation est aisée dès lors qu'un minimum de données sont disponibles ou facilement estimables, puisqu'il s'agit uniquement d'anticiper les situations et de les analyser sur la base de calculs simples. L'argument de la difficulté technique paraît peu recevable et il est même possible d'avancer que la démarche ALARA est, d'ores et déjà, mise en cuvre de façon "pragmatique" dans certaines installations des secteurs industriel et médical.

A titre d'exemple, la démarche mise en œuvre par les laboratoires Pasteur Mérieux lors de la création d'un nouveau laboratoire répond tout à fait à l'approche ALARA. Le point de départ de cette démarche était la nécessité d'améliorer certains éléments, essentiellement dans l'organisation du travail et la conception des équipements dans un laboratoire de recherche. En 1992, la création d'un nouveau laboratoire destiné à effectuer des tests sérologiques dans le cadre d'essais cliniques, et où allaient coexister risque radiologique et exposition à des agents viraux, fut décidée dans un bâtiment existant où aucune substance radioactive n'était encore manipulée. L'activité prévisible consistait en des marquages et des contrôles (le principal radioélément manipulé étant l'iode 125). Les étapes de la démarche de prévention mise en œuvre par PasteurMérieux furent : tout d'abord, la formation d'une personne compétente (la responsable du laboratoire), ensuite la réalisation, par cette responsable en collaboration avec les maîtres d'ouvrage, d'un dossier préparatoire à la conception, incluant les prescriptions techniques, les options de radioprotection...

Ce dossier fut soumis pour avis et conseil à la section de radioprotection des installations du Centre d'études nucléaires de Saclay. Cette prise de conseil permit de corriger de façon prévisionnelle certains des paramètres prévus concernant la filtration, les éléments de protection biologique, de définir l'emplacement des zones en tenant compte des activités adjacentes au laboratoire, de connaître les adresses de fabricants adaptés aux besoins, d'aider à la rédaction des autorisations... Ainsi furent choisis précisément les équipements de protection technique collective : filtration en cascade, utilisation des boîtes à gants pour les marquages, appareils de contrôle in situ, douches, sas d'entrée, modalités d'évacuation et de traitement des déchets... Des contrôles de débit et d'étanchéité des équipements de protection furent réalisés par des sociétés spécialisées.

Une journée de formation relative aux risques spécifiques du laboratoire fut organisée par l'Institut national des sciences et techniques nucléaires (INSTN) in situ pour l'ensemble des agents du laboratoire. La démarche fut alors d'intégrer et de responsabiliser les opérateurs du laboratoire avant tout début de manipulation, en rédigeant ensemble les protocoles de manipulation, en pre- 
nant en compte les exigences de chaque poste de travail. Tous ces protocoles furent analysés en groupe dans le cadre d'une stratégie d'assurance de qualité globale considérant pas à pas les différentes étapes : définition des zones, utilisation des sources, gestion et suivi des déchets, contrôles à effectuer et gestion optimale des temps de présence en laboratoire chaud (8 personnes devant manipuler sur 3 postes de travail en zone contrôlée). Il fut également décidé par un protocole spécial qu'une formation spécifique serait assurée par la personne compétente pour tout travailleur d'une entreprise extérieure pouvant avoir à intervenir dans ce laboratoire.

On retrouve bien ici de nombreux éléments d'une démarche ALARA :

- volonté politique de prévention,

- intégration de la radioprotection et de la gestion des risques à côté de la maîtrise d'œuvre dès la conception d'une installation,

- intégration de la radioprotection et de la gestion des risques lors de l'élaboration des procédures d'exploitation,

- formation et motivation de tous les acteurs,

- utilisation du retour d'expérience extérieur au laboratoire,...

Dans cet exemple, la démarche mise en œuvre a nécessité des moyens financiers et humains, que Pasteur-Mérieux a estimé raisonnables, sans pour autant faire appel explicitement à un système de valeurs monétaires de référence d'unité de risques évités ou à des techniques d'optimisation complexes. Une telle démarche pourrait, dans de nombreux cas, être encore plus simple et ne faire appel qu'au bon sens qui permet, entre autres, d'éviter les expositions inutiles, étape fondamentale de toute démarche ALARA.

\section{La recherche des priorités}

Le secteur électronucléaire ne comporte que quelques dizaines d'installations et il est relativement aisé de suivre les expositions individuelles et collectives correspondant à leurs activités. Dans les autres secteurs, le nombre de sources est beaucoup plus important et les centres de décision sont morcelés. Les 104000 travailleurs exposés du milieu médical sont, en effet, répartis dans plus de 15000 établissements ou cabinets utilisant environ 60000 appareils début 1993 (annexe A). Dans le secteur industriel, les autorisations de détention de radioéléments artificiels sont passées de l'ordre de 1800 à plus de 8 000 entre les années 1972 et 1992, et le nombre d'appareils utilisant des radioéléments dépasse légèrement les 15000 en 1992 pour un peu moins de 25000 travailleurs exposés répartis dans plus de 3000 établissements, pour la plupart des PME ou PMI [5] (annexe A). Face à un tel émiettement, les personnes responsables de la prévention de risques (personnes compétentes, ingénieurs de sécurité, médecins du travail,...) sont bien souvent isolées, mal informées (surtout dans les activités où le process industriel est totalement étranger aux 
rayonnements), et ne savent pas comment aborder le problème de l'optimisation. Il convient donc de tenter de leur simplifier le travail en définissant clairement les priorités.

Cette simplification est envisageable de deux façons :

- par la définition de niveaux d'investigation par type d'activité en termes d'expositions individuelles annuelles pour caractériser les populations qui justifient prioritairement d'une étude d'optimisation ; ces populations prioritaires doivent être celles qui reçoivent des expositions considérées comme élevées pour chaque type de pratique, puisque le processus d'optimisation doit viser à réduire en priorité les expositions des populations les plus exposées (CIPR 60 [12]) ;

- par la réalisation d'études d'optimisation dans des centres de référence, pour des examens ou des pratiques spécifiques, en particulier pour toutes les nouvelles pratiques telles que la radiologie interventionnelle ; ces études permettraient de fournir aux autres entreprises, services ou cabinets des informations sur ce que pourrait être une pratique optimisée, dans des conditions de référence, en matière de radioprotection.

\subsection{L'approche du NCRP}

Des propositions ont été effectuées aux Etats-Unis dans le rapport $107 \mathrm{du}$ National council on radiation protection and measurements (NCRP) [9] pour fixer des niveaux d'investigation dans le secteur médical, mais la démarche et ses limites seraient les mêmes dans le secteur industriel. Retenons que le NCRP estime prioritaires les services ou cabinets pour lesquels certaines doses individuelles sont soit supérieures à la dose individuelle au-dessous de laquelle se situe un pourcentage très important des travailleurs exposés $(80,90 \%)$ dans l'ensemble du secteur soit, à défaut, supérieures à la dose équivalente moyenne des travailleurs exposés de l'ensemble du secteur. En utilisant l'une de ces règles, la personne responsable de la radioprotection pourrait comparer les valeurs d'exposition fournies par les films dosimètres des services dont elle a la charge à ces niveaux de référence et, ainsi, situer les urgences. Dans cette optique, le tableau V fournit des informations disponibles à partir des statistiques publiées par le SCPRI pour différents types d'activités médicales.

Si l'on fait l'hypothèse que l'urgence en matière d'optimisation concerne tous les services où des travailleurs dépassent la dose en dessous de laquelle se situent $90 \%$ des travailleurs exposés dans le secteur, les statistiques disponibles permettraient de considérer que $5 \mathrm{mSv}$ correspond à ce niveau d'investigation pour le radiodiagnostic et la radiothérapie externe, c'est-à-dire que tout service où des travailleurs exposés dépasseraient les $5 \mathrm{mSv}$ annuels devrait faire l'objet d'une investigation prioritaire en matière d'optimisation. Pour les autres types d'activités, les statistiques n'étant pas suffisamment fines pour déterminer ce seuil de $90 \%$ et pour suivre la règle proposée par le NCRP, il conviendrait de retenir la dose moyenne comme niveau d'investigation. 
TABLEAU V

Dose individuelle moyenne et pourcentage de travailleurs exposés à moins de $5 \mathrm{mSv}$ selon le type d'activité dans le domaine médical en 1993 Mean individual doses and workers (\%) exposed to less than $5 \mathrm{mSv}$ according to their medical activities (1993)

\begin{tabular}{|c|c|c|}
\hline Activité & indiv & $\begin{array}{l}\text { urs exposés } \\
\text { de } 5 \text { mSv } \\
0 \%\end{array}$ \\
\hline Radiodiagnostic & 3,2 & 91 \\
\hline Dentaire & 2,0 & 95 \\
\hline Radiothérapie externe & 3,2 & 88 \\
\hline Curiéthérapie & 3,5 & 96 \\
\hline Médecine nucléaire in vivo & 2,0 & 94 \\
\hline Médecine nucléaire in vitro & 1,6 & 100 \\
\hline
\end{tabular}

Une telle approche permet, sans doute, de réduire certaines expositions, mais se heurte rapidement au caractère grossier des données statistiques et, en tout état de cause, ne prouve nullement qu'en dessous du niveau d'investigation l'optimum soit atteint : l'exemple du radiodiagnostic illustrera le premier point et celui du dentaire le second.

L'application des rayons $X$ au radiodiagnostic couvre un large domaine de procédures (radiographie conventionnelle, mammographie, scanographie, angiographie, radiologie interventionnelle,...) dont la mise en œuvre conditionne directement l'exposition professionnelle des travailleurs. En effet, on peut distinguer dans ces procédures celles où l'opérateur est à distance du patient (source principale de rayonnement diffusé), protégé par un paravent en plomb et verre plombé, et celles où l'opérateur est à proximité du patient, et où sa protection partielle est assurée par le port d'un tablier ou d'accessoires (lunettes, cache-thyroïde,...). Dans ce deuxième cas, le débit de dose dans l'air peut excéder $2 \mathrm{mGy} / \mathrm{h}$ et les procédures peuvent durer plusieurs dizaines de minutes. Il convient également de souligner les procédures particulières, telles que celles pratiquées en radiologie interventionnelle où les niveaux d'exposition sont beaucoup plus élevés et où la dosimétrie film ne rend pas compte de l'exposition véritable. Pour ces diverses raisons, retenir un seuil unique de $5 \mathrm{mSv}$ comme seuil d'investigation pour l'ensemble du radiodiagnostic n'aurait que peu de signification. La logique précédente amènerait à retenir pour les procédures où l'opérateur est à distance un niveau nettement plus faible, mais 
ne serait pas du tout adaptée à des situations comme celle de la radiologie interventionnelle. La règle proposée par le NCRP n'apparaît donc pas pertinente pour déterminer les priorités.

Le secteur dentaire se caractérise, pour sa part, par un nombre élevé d'installations et un faible risque d'exposition, dû principalement au diffusé par le patient. La protection est assurée par l'appareillage lui-même, où le rayonnement de fuite est minime, par l'éloignement de l'opérateur et/ou par l'utilisation d'écrans. Le tableau V montre que la dose moyenne "corps entier" est de $2 \mathrm{mSv}$ pour les travailleurs exposés. Bien que cette valeur soit faible, les conditions de travail sont telles que l'application des règles de base de la radioprotection devraient normalement conduire systématiquement à des niveaux d'exposition inférieurs au seuil de mesure $(0,20 \mathrm{mSv})$. Une approche analytique simple et non statistique montre qu'à coup sûr, dès lors qu'une exposition individuelle devient significative dans ce secteur, on est "loin" de l'optimum. La règle proposée par le NCRP n'apparaît donc pas, ici non plus, pertinente.

Il ressort, cependant, des exemples analysés qu'il serait nécessaire d'adapter les statistiques à la réalité pour mieux la cerner. On peut, ainsi, envisager notamment de modifier la typologie retenue ou de compléter les données statistiques par des enquêtes sur échantillon, mais cela ne permettra pas, de toutes façons, de déterminer de véritables seuils d'investigation.

\subsection{Les études génériques}

La consultation d'équipes d'experts au niveau national serait souhaitable pour proposer des pratiques optimisées à partir de l'analyse de services ou d'entreprises jugés représentatifs. A cette étape, l'approche analytique s'appuyant d'une façon ou d'une autre sur une dosimétrie opérationnelle, même non "sophistiquée", et de véritables études d'optimisation, sera inévitable. Il sera donc nécessaire de s'adapter aux différentes utilisations des sources en milieu médical ou industriel afin d'analyser les postes, de définir des protections adaptées et d'apprécier l'apport de ces mesures en terme de réduction des doses collectives et individuelles. Les résultats de ces études permettront de fournir des informations sur les doses collectives optimisées par type de tâches et sur les doses individuelles correspondantes en fonction de niveaux d'activités. On pourra, alors, envisager de définir des contraintes de doses au sens de la Publication CIPR 60 [12]. La personne responsable pourra alors situer son (ses) service(s) et juger s'il convient ou non d'engager des efforts pour se rapprocher de cette pratique optimisée.

\section{Les vraies difficultés}

Mettre en œuvre l'optimisation suppose une gestion a priori des expositions. Cela revient à dire que l'on est capable d'anticiper et de prévoir où, quand, comment et par qui seront prises les expositions correspondant aux 
tâches envisagées [5]. Une telle anticipation suppose soit que l'on ait des données fines de retour d'expérience sur les expositions liées à ces tâches, soit que l'on sache estimer les sources, les temps de travail exposé, et l'impact des conditions dans lesquelles le travail est effectué. Dans les secteurs médical et industriel non électronucléaire, une telle anticipation est rendue particulièrement délicate lorsque la seule dosimétrie individuelle est celle obtenue par le film dosimètre réglementaire, et que les mesures d'ambiance sont peu pratiquées. L'absence de données adéquates correspond donc à un vrai problème pour mettre en œuvre l'optimisation.

Les responsables de l'électronucléaire se sont heurtés, il y a quelques années, à ce problème qui a trouvé progressivement une solution avec la mise en place de systèmes de dosimétrie opérationnelle. Un tel système fait actuellement son apparition dans certains services hospitaliers [2]. Cependant, il convient d'insister sur le fait qu'une "dosimétrie opérationnelle sophistiquée", dont la mise en place est coûteuse et prend du temps, n'est pas le seul moyen pour recueillir les informations nécessaires à l'optimisation : l'expérience montre qu'en l'absence de données fines, il est toujours possible de faire appel aux hommes de terrain pour estimer des expositions liées à des tâches et calculer l'impact d'actions de protection envisageables ; un tel retour d'expérience semble parfaitement applicable aux secteurs industriel et médical ainsi qu'en témoignent certains exposés présentés lors de ce congrès $[1,18]$.

Rester dans la seule logique du respect du principe de limitation de dose est le second problème (cf. $\S 1.4$ ), qui, il faut le rappeler, constitue le principal frein à la mise en œuvre de l'optimisation. Dans cette logique, respecter la limite apparaît tout à fait suffisant, a fortiori dans une situation où les expositions lui sont largement inférieures. Le vrai problème consiste donc à faire sortir de cette logique les acteurs des secteurs industriel et médical : responsables d'entreprises, médecins, préparateurs, travailleurs, personnes compétentes, gestionnaires,... Pour cela, il est indispensable de promouvoir l'information sur la notion de risque résiduel aux faibles expositions, sur la volonté de prudence qui sous-tend toute la démarche de l'optimisation et sur la démarche qui conduit à établir des niveaux de risques résiduels acceptables. Le retour d'expérience montre que l'information sur ces notions est bien perçue par les personnes de terrain et qu'elle ne crée aucun effet de peur ou d'anxiété. Bien au contraire, elle responsabilise chacun et l'incite à participer à la gestion du risque radiologique.

Cette information, décidée par la hiérarchie, n'a certes pas encore abouti à une généralisation complète de la culture du risque résiduel dans le domaine électronucléaire, mais il a suffi de quelques années pour que cette culture soit largement répandue. Cependant, dans les secteurs industriel et médical, les travailleurs exposés sont répartis, le plus souvent, en petit nombre sur de nombreux établissements. Il en résulte une dispersion de responsabilités pour la radioprotection des travailleurs et des difficultés pour le suivi des effets de mesures de radioprotection. Il paraît peu probable que la hiérarchie s'engage 
sur la gestion du risque radiologique lorsque celui-ci est complètement marginal par rapport à l'activité première de l'entreprise. Les personnes compétentes et les médecins du travail apparaissent alors les acteurs privilégiés dans cette diffusion de la culture du risque résiduel, car ce sont eux qui peuvent réellement enclencher la démarche ALARA. On peut aussi ajouter que le problème de la diffusion de la culture du risque résiduel ne sera vraiment en voie d'être résolu que lorsque le grand public lui même y sera sensibilisé.

\section{Conclusion}

Il apparaît ainsi que les vraies difficultés pour la mise en œuvre de l'optimisation dans les secteurs industriel et médical ne sont pas celles le plus souvent mises en avant (faibles niveaux d'exposition, ressources insuffisantes, complexité,...), mais plutôt un manque de données appropriées et, surtout, une absence de culture du risque résiduel. Une volonté politique claire des instances concernées, tant au niveau national qu'au niveau des entreprises, est nécessaire pour lever assez rapidement de tels obstacles, comme cela a été le cas depuis quelques années dans le secteur électronucléaire. L'existence de ces freins n'empêche cependant pas la mise en œuvre de la démarche ALARA en s'appuyant sur les acteurs privilégiés : personnes compétentes, médecins du travail. Le cheminement de cette démarche implique de s'attaquer à de nombreux problèmes que l'on ne fera qu'évoquer dans cette conclusion tels que : la mise en place d'une dosimétrie réellement représentative que ce soit au corps entier ou à certains organes (thyroïde, extrémités,...), par exemple dans le cas des personnels vétérinaires ou de radiologie interventionnelle; la présence du patient dans le secteur médical qui soulève le problème des transferts de risques entre groupes sociaux... En pratique, c'est le développement d'un "état d'esprit" de coopération et de responsabilisation de l'ensemble des acteurs face au risque radiologique qui permettra une mise en œuvre effective du principe d'optimisation dans les secteurs industriel et médical.

\section{Annexe A}

\section{Données détaillées concernant les expositions dans les secteurs médical et industriel non électronucléaire}

Un panorama de l'exposition professionnelle en 1993, selon les principales utilisations médicales des rayonnements ionisants pour les travailleurs du milieu médical surveillés par le seul SCPRI, est présenté dans les tableaux A.I et A.II.

Un panorama de l'exposition professionnelle en 1993 est présenté dans le tableau A.III pour l'ensemble des travailleurs surveillés par le SCPRI, et par le LCIE en ce qui concerne les travailleurs des industries non électronucléaires et la recherche. Le premier élément notable dans ce tableau est la part impor- 
TABLEAU A.I

Exposition professionnelle des travailleurs du secteur médical surveillés par le SCPRI en 1993 (source SCPRI)

Occupational exposure of medical workers (SCPRI monitoring, 1993)

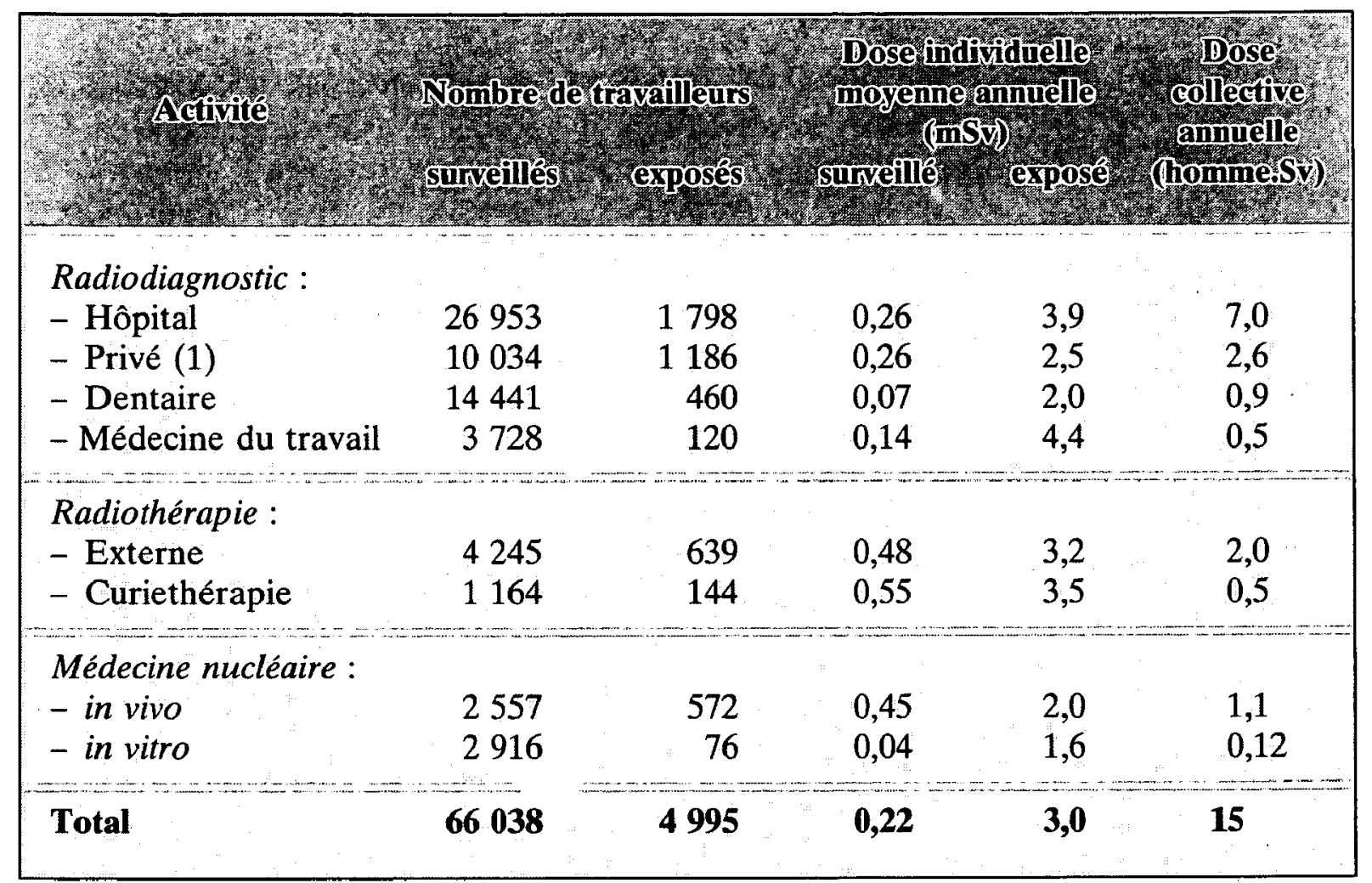

(1) Médecine générale privée + médecine spécialisée et clinique + cabinets de radiologie privées.

TABLEAU A.II

Parc radiologique médical français en 1993, d'après les statistiques du SCPRI Medical installations in France, 1993 (SCPRI data)

\begin{tabular}{|lr|}
\hline \multicolumn{1}{|c|}{ Type dinstallation } & 1993 \\
\hline Radiodiagnostic : & \\
- radioscopie & 1894 \\
- radiodiagnostic léger & 3700 \\
- radiodiagnostic lourd & 12955 \\
- mammographie & 2101 \\
- scanographie & 447 \\
- dentaire & 35504 \\
Radiothérapie : & \\
- accélérateurs & 192 \\
- cobalthérapie & 167 \\
- curiethérapie & 119 \\
Médecine nucléaire : & \\
- caméra à scintillations & 252 \\
\hline
\end{tabular}


TABLEAU A.III

Bilan 1993 de l'exposition professionnelle des travailleurs du secteur industriel non électronucléaire et de la recherche (source SCPRI)

Occupational exposure of workers in the industrial non-electronuclear field and in research (SCPRI data)

\begin{tabular}{|c|c|c|c|c|c|}
\hline \multicolumn{3}{|c|}{ 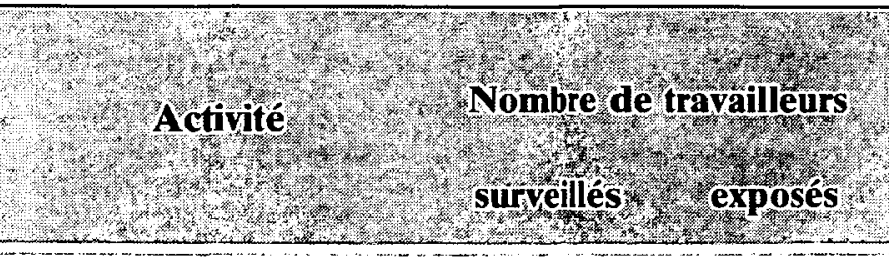 } & \multicolumn{3}{|c|}{ 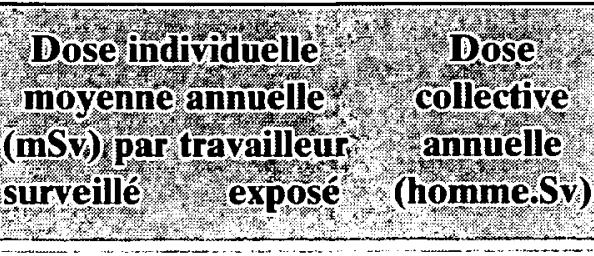 } \\
\hline $\begin{array}{l}\text { Radiographie et } \\
\text { gammagraphie } \\
\text { industrielle }\end{array}$ & 1496 & 176 & 0,45 & 3,8 & 0,67 \\
\hline $\begin{array}{l}\text { Sources non scellées, } \\
\text { industrie + recherche }\end{array}$ & 4565 & 214 & 0,09 & 1,9 & 0,42 \\
\hline $\begin{array}{l}\text { Autres applications } \\
\text { non médicales }\end{array}$ & 5702 & 680 & 0,42 & 3,6 & 2,4 \\
\hline Non ventilées & 15420 & 1403 & 0,65 & 7,15 & 10,03 \\
\hline $\begin{array}{l}\text { Total industrie } \\
\text { non nucléaire }\end{array}$ & 27183 & 2473 & $\mathbf{0 , 5 0}$ & 5,47 & 13,52 \\
\hline Vétérinaires & 1898 & 121 & 0,14 & 2,2 & 0,27 \\
\hline
\end{tabular}

TABLEAU A.IV

Répartition des appareils utilisant les rayonnements ionisants, par type d'appareils dans l'industrie non électronucléaire ${ }^{1}$

Distribution of devices using ionising radiation in the non-electronuclear industry

\begin{tabular}{|lr|}
\hline Rayons X industrie & 785 \\
Rayons X vétérinaire & 33 \\
Accélérateurs & 16 \\
& \\
Irradiateurs (hors INB, hors poche de sang) & 80 \\
Irradiateurs (INB) & 5 \\
Gammagraphes & 850 \\
Densité, pesage, niveau & 7700 \\
Epaisseur & 2700 \\
Niveau d'embouteillage & 260 \\
Niveau de gaz & 180 \\
Humidimètre, densimètre (chantiers) & 1100 \\
Analyseurs d'alliage (fluo X) & 200 \\
Détecteurs à capture d'électrons & 1400 \\
\hline
\end{tabular}

1. D'après VIDAL H. - Les rayonnements ionisants, applications médicales et industrielles. Journées SFRP "Sécurité des sources et de rayonnements ionisants", Saclay, 9-10 juin 1993. Radioprotection, 1994, 29 (2) 223-229. 
TABLEAU A.V

Principaux utilisateurs de radioéléments

(nombre d'autorisations de détention en 1992)

Main radionuclide users (number of licenses, 1992)

\begin{tabular}{|c|c|}
\hline \multicolumn{2}{|c|}{$\begin{array}{l}\text { Utilisations (en sourres non scellees) } \\
2 \mathrm{e} \text { section (industifulle) }\end{array}$} \\
\hline $\begin{array}{l}\text { Recherche } \\
\text { Etalonnage et enseignement }\end{array}$ & $\begin{array}{l}603 \\
107\end{array}$ \\
\hline \multicolumn{2}{|c|}{$\begin{array}{l}\text { Utilisations (en sources scellees) } \\
\text { 2e section (industrielle) }\end{array}$} \\
\hline Gammagraphie & 302 \\
\hline Irradiateurs & 52 \\
\hline Mesure de densité et pesage & 635 \\
\hline Mesure d'épaisseur & 900 \\
\hline Mesure de niveau & 705 \\
\hline Mesure d'humidité & 186 \\
\hline Mesure d'humidité + densité & 331 \\
\hline Elimination d'électricité statique & 60 \\
\hline Chromatographie en phase gazeuse & 536 \\
\hline Analyses diverses & 403 \\
\hline Etalonnage et enseignement & 567 \\
\hline Total des utilisateurs & 5387 \\
\hline
\end{tabular}

Une autorisation peut couvrir plusieurs types d'utilisation.

tante que représentent les activités non ventilées (LCIE) et les "autres applications non médicales", avec plus de 21000 travailleurs surveillés, soit plus des trois quarts de l'ensemble du secteur industriel.

Dans le secteur industriel non électronucléaire, le SCPRI suit 11763 travailleurs et le LCIE 15420. En appliquant le ratio "travailleurs significativement exposés/travailleurs surveillés" de la population suivie par le SCPRI à celle suivie par le LCIE, on peut estimer la dose moyenne annuelle par personne exposée à 3,26 mSv pour la population suivie par le SCPRI et à 7,15 mSv pour celle suivie par le LCIE.

\section{Annexe B}

\section{Enquête du ministère du travail sur les pratiques dans la région Aquitaine}

Une enquête sur les rayonnements ionisants, réalisée par sondage dans la région Aquitaine, a été publiée dans le Bilan annuel sur les conditions de travail du Conseil supérieur de prévention des risques professionnels en 1993 [6]. 
Cette enquête a porté sur 128 entreprises (64 services ou cabinets médicaux, dentaires ou vétérinaires et 64 entreprises du secteur industriel) sélectionnées à partir des fichiers de la CIREA et du CAMARI.

Le tableau B.I résume, pour les différents secteurs suivis, le nombre d'entreprises déclarant des travailleurs de catégorie A (personnes directement affectées à des travaux sous rayonnements, et dont les conditions de travail sont susceptibles d'entraîner le dépassement des 3/10 des limites annuelles) et, pour ceux-ci, le nombre d'entreprises les faisant suivre médicalement et dosimétriquement.

\section{TABLEAU B.I}

Pratiques des entreprises face à la réglementation en matière de radioprotection [6] Industry pratices toward regulation in radiation protection

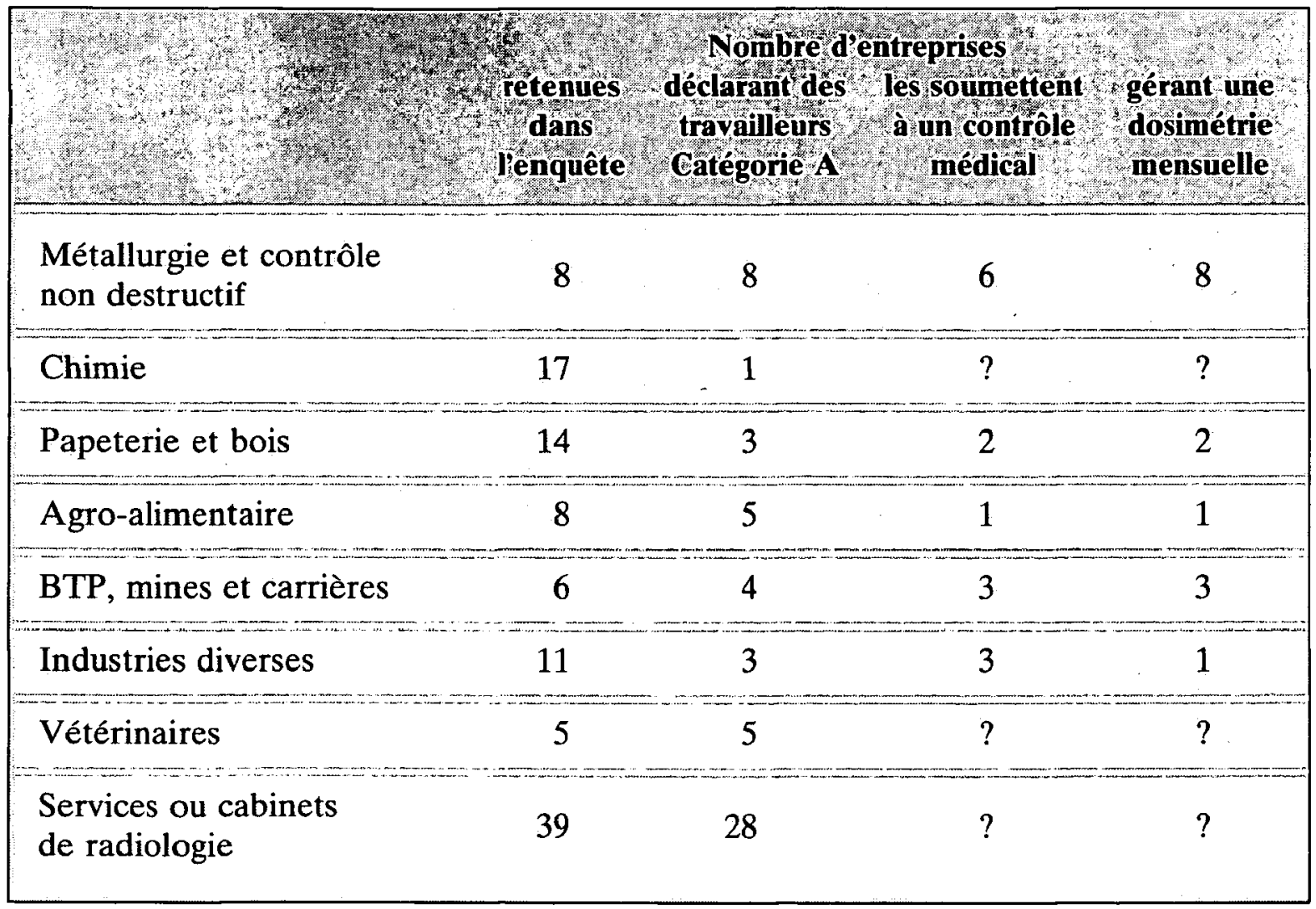

\section{Remerciements}

Nous tenons à remercier tout particulièrement MM. Dutreil, Chanteur et Biau du SCPRI pour l'aide qu'ils nous ont fournie dans la collecte des informations statistiques concernant les secteurs industriel et médical ; MM. Hubert, Dagorn et Bergot pour le groupe CEA; MM. Dollo et Daubert pour EDF.

Par ailleurs, nous tenons à remercier M. Paturel et Mme Blondeau de Pasteur-Mérieux, Val de Reuil, qui nous ont permis d'utiliser l'expérience de leurs laboratoires et nous ont fourni, en collaboration avec $M$. Orts du SPR CE-Saclay, les informations nécessaires. 


\section{RÉFÉRENCES}

[1] ARTHUS J.C. - Radioexposition de l'entourage d'un patient traité par iode 131. Journées SFRP sur "L'optimisation de la radioprotection des travailleurs dans les domaines électronucléaire, industriel et médical", La Rochelle, 20-21 septembre 1994.

[2] AUBERT B., LAMON A., BEAUDET G. - Evaluation des nouveaux dosimètres électroniques en milieu hospitalier. Journées SFRP sur "L'optimisation de la radioprotection des travailleurs dans les domaines électronucléaire, industriel et médical", La Rochelle, 20-21 septembre 1994.

[3] BLAIN A., JUPPET P. - Le porteur ARAMIS : analyse coût-efficacité dans un contexte ALARA. Journées SFRP sur "L'optimisation de la radioprotection des travailleurs dans les domaines électronucléaire, industriel et médical", La Rochelle, 2021 septembre 1994.

[4] COMMISSARIAT A L'ENERGIE ATOMIQUE (CEA), INSTITUT DE PROTECTION ET DE SURETE NUCLEAIRE (IPSN). - Statistiques d'exposition du personnel, rapport provisoire. Fontenay-aux-Roses : IPSN, 1993.

[5] D'ASCENZO L. - Optimisation de la radioprotection et gestion des expositions individuelles. Journées SFRP sur "L'optimisation de la radioprotection des travailleurs dans les domaines électronucléaire, industriel et médical", La Rochelle, 2021 septembre 1.994.

[6] CONSEIL SUPERIEUR DE LA PREVENTION DES RISQUES PROFESSIONNELS. - Enquête sur les rayonnements ionisants. Conditions de travail, bilan 193. Paris : Ministère du travail, de l'emploi et de la formation professionnelle, 1993, chap. 9, 333-357.

[7] ELECTRICITE DE FRANCE (EDF). - Sécurité et radioprotection, bilan 1993. Saint-Denis : EDF/DSRE, 1993.

[8] ELECTRICITE DE FRANCE (EDF). - Livre blanc sur la radioprotection. Paris : EDF, 1993.

[9] ETATS-UNIS. NATIONAL COUNCIL ON RADIATION PROTECTION AND MEASUREMENTS (NRCP). - Implementation of the principle of as low as readily achievable (ALARA) for medical and dental personnel (NRCP report $\mathbf{n}^{\circ} 107$ ). Washington : NRCP, 1990.

[10] FRANCE. - Décret 86-1103 du 2 octobre 1986 relatif à la protection des travailleurs contre les dangers des rayonnements ionisants hors des installations nucléaires de base. In : Protection contre les rayonnements ionisants (Brochure $\mathrm{n}^{\circ} 1420$ ). Paris : Journal officiel, 1990, 2, 427-510.

[11] FRANCE. - Décret 88-662 du 6 mai 1988 relatif à la protection des travailleurs contre les dangers des rayonnements ionisants dans des installations nucléaires de base. In : Protection contre les rayonnements ionisants (Brochure ${ }^{\circ}{ }^{\circ} 1420$ ). Paris : Journal officiel, 1990, 2, 513-558.

[12] INTERNATIONAL COMMISSION ON RADIOLOGICAL PROTECTION (ICRP). - Implications of the Commission's recommendations that dose be kept as low as readily achievable (ICRP Publication 22). Oxford : Pergamon press, 1973.

[13] INTERNATIONAL COMMISSION ON RADIOLOGICAL PROTECTION (ICRP). - Recommendations 1990... (ICRP Publication 60). Oxford : Pergamon press, 1993. (ed. française, 1993).

[14] LEFAURE C., LOCHARD J., SCHNEIDER T., SCHIEBER C. - Proposition pour un système de valeurs monétaires de référence de l'homme-sievert. Rapport CEPNR-193, 1993.

[15] LOCHARD J. - Le développement du principe ALARA en France. Journées SFRP sur "L'optimisation de la radioprotection des travailleurs dans les domaines électronucléaire, industriel et médical", La Rochelle, 20-21 septembre 1994. 
[16] LOCHARD J., MACCIA C., PAGES P. - La valeur implicite de l'homme-rem travailleur. Analyse de quelques mesures adoptées sur les PWR français. Rapport CEPN-R-45, 1981.

[17] MACCIA C., LOCHARD J. - Evaluation de la protection collective du personnel d'un service de radiologie. Rapport CEPN-R-57, 1982.

[18] PAUWELS N. , ClAPIER F., CIEUR M., GENet S., PROUST J., MAZAL A., BOUCAULT JC., DELACROIX S., FERRAND R., HABRAND JL., LOUIS M., NAURAYE C. - Optimisation de la radioprotection des travailleurs au centre de protonthérapie d'Orsay. Journées SFRP sur "L'optimisation de la radioprotection des travailleurs, dans les domaines électronucléaire, industriel et médical", La Rochelle, 20-21 Septembre 1994.

[19] SERVICE CENTRAL DE PROTECTION CONTRE LES RAYONNEMENTS IONISANTS (SCPRI). - Communication personnelle, juin 1994. 